\title{
Efeitos da nutrição materna na gestação sobre a qualidade da progênie - uma revisão
}

\author{
Effects of maternal nutrition on pregnancy on progeny quality - a review \\ Efectos de la nutrición materna en la preñez sobre la calidad de la progênie - una revisión
}

Recebido: 04/02/2021 | Revisado: 12/02/2021 | Aceito: 15/02/2021 | Publicado: 23/02/2021

\author{
John Lenon Klein \\ ORCID: https://orcid.org/0000-0001-8337-4152 \\ Universidade Federal de Santa Maria, Brasil \\ E-mail: johnlenonklein@gmail.com \\ Diego Soares Machado \\ ORCID: https://orcid.org/0000-0002-2406-280X \\ Universidade Federal de Santa Maria, Brasil \\ E-mail: dsm_zootecnista@hotmail.com \\ Sander Martinho Adams \\ ORCID: https://orcid.org/0000-0003-4895-8237 \\ Universidade Federal de Santa Maria, Brasil \\ E-mail: sander.adams@hotmail.com \\ Dari Celestino Alves Filho \\ ORCID: https://orcid.org/0000-0003-2559-7504 \\ Universidade Federal de Santa Maria, Brasil \\ E-mail: darialvesfilho@hotmail.com \\ Ivan Luiz Brondani \\ ORCID: https://orcid.org/0000-0002-6526-3042 \\ Universidade Federal de Santa Maria, Brasil \\ E-mail: ivanbrondani@gmail.com
}

\begin{abstract}
Resumo
O objetivo do estudo foi, por meio de uma revisão de literatura, avaliar os efeitos da nutrição de vacas de corte durante a gestação sobre a qualidade e o desempenho da progênie após o nascimento. Nos últimos anos, inúmeras pesquisas foram desenvolvidas para avaliar os efeitos da nutrição materna durante a gestação (programação fetal) sobre a produtividade da progênie. Diante dos resultados apresentados, está claramente elucidado que as condições nutricionais do útero grávido alteram de várias maneiras a saúde, a fisiologia, o metabolismo e, consequentemente, $o$ desempenho pós-parto da progênie. Estas alterações podem variar de acordo com o grau e intensidade do desafio nutricional, momento deste desafio no decorrer da gestação e capacidade adaptativa das vacas gestantes em particionar os nutrientes ao feto. A literatura sugere que os efeitos da programação fetal são mais facilmente constatados nos meses iniciais de vida dos descendentes, desaparecendo com o avançar da idade, porém, a restrição nutricional da vaca durante a gestação também pode levar à formação de um indivíduo com maior capacidade de adaptação e mais preparado para sobreviver em ambientes mais desafiadores na vida pós-natal. Além disso, a formação de um fenótipo "econômico" poderá resultar em um desempenho compensatório durante a vida adulta dos bezerros de vacas que passaram por desafios nutricionais durante a gestação.
\end{abstract}

Palavras-chave: Miogênese; Músculo esquelético; Novilhos; Programação fetal; Bovinos de corte.

\begin{abstract}
The aim of the study was, through a literature review, to evaluate the effects of nutrition of beef cows during pregnancy on the quality and performance of the progeny after birth. In recent years, numerous studies have been carried out to assess the effects of maternal nutrition during pregnancy (fetal programming) on progeny productivity. In view of the results presented, it is clearly elucidated that the nutritional conditions of the pregnant uterus alter health, physiology, metabolism and, consequently, the postpartum performance of the progeny in several ways. These changes may vary according to the degree and intensity of the nutritional challenge, the moment of this challenge during pregnancy and the adaptive capacity of pregnant cows to partition nutrients to the fetus. The literature suggests that the effects of fetal programming are more easily seen in the initial months of the offspring's life, disappearing with advancing age, however, the nutritional restriction of the cow during pregnancy can also lead to the formation of an individual with greater capacity to adaptation and better prepared to survive in more challenging environments in postnatal life. In addition, the formation of an "economic" phenotype may result in a compensatory performance during the adult life of cow calves that have undergone nutritional challenges during pregnancy.
\end{abstract}

Keywords: Beef cattle; Fetal programming; Myogenesis; Skeletal muscle; Steers. 


\section{Resumen}

El objetivo del estudio fue, a través de una revisión de la literatura, evaluar los efectos de la nutrición de las vacas de carne durante la gestación sobre la calidad y desempeño de la progenie después del nacimiento. En los últimos años se han realizado numerosos estudios para evaluar los efectos de la nutrición materna durante la preñez (programación fetal) sobre la productividad de la progenie. A la vista de los resultados presentados, se aclara claramente que las condiciones nutricionales del útero gestante alteran la salud, fisiología, metabolismo y, en consecuencia, el desempeño posparto de la progenie de varias formas. Estos cambios pueden variar según el grado y la intensidad del desafío nutricional, el momento de este desafío durante la preñez y la capacidad de adaptación de las vacas gestantes para repartir los nutrientes al feto. La literatura sugiere que los efectos de la programación fetal se ven más fácilmente en los primeros meses de vida de la descendencia, desapareciendo con el avance de la edad, sin embargo, la restricción nutricional de la vaca durante la gestación también puede conducir a la formación de un individuo con mayor capacidad para adaptación y mejor preparada para sobrevivir en entornos más desafiantes en la vida posnatal. Además, la formación de un fenotipo "económico" puede resultar en un desempeño compensatorio durante la vida adulta de los terneros que han sufrido desafíos nutricionales durante la preñez.

Palabras clave: Miogénesis; Músculo esquelético; Novillos; Programación fetal; Ganado vacuno.

\section{Introdução}

Aumentar a eficiência produtiva de bovinos de corte e, consequentemente, elevar a oferta de produtos cárneos é essencial para atender as demandas cada vez maiores por estes produtos alimentícios de alto valor biológico. Estudos recentes vêm aprofundando os conhecimentos sobre os fatores pré-natais que podem alterar o potencial produtivo da progênie na vida adulta, isto é, condições uterinas durante a gestação, estes processos são conhecidos como programação fetal (Mendes, 2016).

Dentre os insultos e desafios ao útero grávido, a nutrição da vaca durante a gestação merece destaque. Em geral, vacas de corte são mantidas em sistemas forrageiros e passam por desafios nutricionais durante a gestação em toda a parte do mundo, uma vez que ocorre priorização da parição destas fêmeas para favorecer a produção de leite e a sobrevivência do neonato. Segundo Du et al. (2013), a ingestão de nutrientes pela vaca gestante está associada à produtividade da prole na vida adulta. Du et al. (2015) afirmaram que filhos de vacas mantidas sob restrita oferta de nutrientes durante a gestação apresentam potencial de produção de carne comprometido, porém, vale destacar que tanto a restrição como o excesso de nutrientes para a vaca durante a gestação podem influenciar a formação fetal e o potencial produtivo da prole. Fornecendo 100 ou $125 \%$ das exigências de nutrientes digestíveis totais no terço final de gestação Wilson et al. (2016) observaram incremento no peso ao nascimento da prole mas sem reflexos no desempenho destes animais na vida adulta.

Vários são os efeitos que a nutrição da vaca na gestação exerce sobre o feto em formação. Adaptações fisiológicas no desenvolvimento fetal foram verificadas desde os primeiros processos de formação fetal após a implantação embrionária, tais como alteração no número de placentomas, vascularização e circulação sanguínea entre a placenta e o útero materno (Camacho et al., 2018). O estabelecimento de uma circulação funcional é fundamental para o desenvolvimento e crescimento fetal (Du et al., 2010). Pesquisas recentes também apontam para interferências da nutrição materna na gestação sobre as características fisiológicas e funcionais de alguns órgãos envolvidos com o metabolismo. Quando testaram dois níveis nutricionais para vacas de corte durante a gestação, Duarte et al. (2013) observaram alterações no intestino delgado de bezerros nascidos de vacas submetidas à alimentação de mantença em relação aos filhos de vacas com alimentação ad libitum.

A nutrição materna durante a gestação pode alterar também a formação do músculo esquelético fetal através dos processos de miogênese e adipogênese fetal (Du et al., 2010), processos que são exclusivos do período gestacional, e quando não favorecidos, acabam comprometendo o potencial produtivo e a qualidade da carne da progênie. Tendo em vista que os fatores nutricionais interferem na formação fetal, esta revisão de literatura tem como objetivo reunir informações referentes aos efeitos da nutrição de vacas de corte durante a gestação sobre a qualidade e a produtividade da progênie na vida pós-natal. 


\section{Nutrição da vaca gestante e a qualidade da progênie}

\subsection{Metabolismo da vaca na gestação}

A produção de bezerros no Brasil é baseada em sistemas forrageiros os quais normalmente apresentam variações sazonais na oferta e na qualidade dos alimentos disponíveis para as vacas de corte. Em geral, as vacas passam a gestação no período de pior oferta forrageira para priorizar a fase de parição e aleitamento dos bezerros, no entanto, estudos recentes têm enfatizado os efeitos prejudiciais da baixa ingestão de nutrientes pela vaca gestante sobre o desenvolvimento futuro da progênie. Maiores demandas nutricionais ocorrem no terço final de gestação, uma vez que nesta fase de gestação, o feto adquire cerca de $75 \%$ do seu peso ao nascimento, sendo estas demandas cerca de $75 \%$ maiores em comparação a uma vaca não gestante de mesmo peso corporal (Bauman \& Currie, 1980). Os mesmos autores comentam ainda que ocorrem maiores demandas fetais por glicose (50 a $70 \%$ dos substratos utilizados pelo feto), consumindo de 30 a $50 \%$ da glicose total ingerida pela vaca.

A partição de nutrientes durante a gestação foi descrita por Bauman e Currie (1980), os quais afirmaram que existem mecanismos endócrinos que regulam sua distribuiçãono organismo animal. Estes autores se basearam nos conceitos de homeorrese e homeostase, sendo esta última definida como a manutenção do equilíbrio fisiológico para manter condições constantes no organismo animal, regulando as funções fisiológicas de forma constante e pré-estabelecidas. Já o conceito de homeorrese está baseado nas mudanças orquestradas nos tecidos para suportar um estado fisiológico de maior prioridade (Bauman \& Currie, 1980).

Alguns fatores homeorreicos descritos na literatura referem-se às mudanças no metabolismo da vaca durante a gestação. Bauman e Currie (1980) descrevem algumas alterações metabólicas que caracterizam os mecanismos homeorreicos, tais como o aumento da secreção de progesterona pelo útero após o reconhecimento materno da gestação, fator que aumenta o fluxo de sangue entre a placenta e o organismo materno e, consequentemente, a drenagem de nutrientes ao feto. Outro aspecto referenciado pelos autores, é a redução da sensibilidade do tecido adiposo à insulina e menor captação de glicose sanguínea para ser armazenada como triglicerídeos. No tecido adiposo, ocorre também aumento na taxa de lipólise e, consequentemente, maior liberação de ácidos graxos livres no sangue (Bauman \& Currie, 1980). Esses mecanismos podem explicar a mobilização de reservas corporais das vacas durante a gestação para manter adequada oferta de nutrientes ao feto em condições moderadas de restrição nutricional (Webb et al., 2019).

A capacidade de adaptação metabólica das vacas e a habilidade de particionar os nutrientes com o feto, pode determinar quão intenso serão os efeitos da nutrição materna durante gestação sobre a formação fetal. Camacho et al. (2018) estudaram os efeitos da restrição alimentar para vacas de corte nos terços iniciais de gestação, e destacam a capacidade de adaptação das vacas multíparas às condições de baixa oferta de nutrientes, bem como a resiliência da fêmea em suprir as demandas fetais. Os autores observaram no referido estudo, aumento no número de placentomas e peso total da placenta em vacas com restrição nutricional, como alternativa para aumentar o suprimento de nutrientes ao feto.

A capacidade de adaptação metabólica também foi descrita por Long et al. (2009), os quais observaram que a restrição nutricional reduziu o peso do feto e dos órgãos vitais aos 125 dias de gestação, porém, destacaram que estas diferenças de crescimento intrauterino desapareceram aos 245 dias de gestação. A suscetibilidade à restrição de nutrientes é maior nas fêmeas mais jovens, ou seja, a partição dos nutrientes para o feto compete com as demandas metabólicas para o crescimento corporal de fêmeas nulíparas (Long et al., 2009). Mohrhauser et al. (2015b) complementam que vacas adultas possuem maiores reservas corporais para atender às demandas fetais em relação a uma novilha jovem, as quais podem ser mais sensíveis à falta de nutrientes durante a gestação.

Da mesma forma que o crescimento corporal das fêmeas jovens compete com o feto, a produção de leite no início da gestação também compete com feto na partição de nutrientes, e pode, consequentemente, alterar o desenvolvimento fetal. 
Gonzales-Recio et al. (2012) observaram que novilhas nascidas de vacas que produziam leite durante a gestação foram metabolicamente menos eficientes, produziram 53 litros a menos de leite e morreram 16 dias antes em relação às filhas de vacas que não produziram leite na gestação. Levando em consideração os fatores discutidos anteriormente, os efeitos da nutrição materna durante a gestação sobre a formação fetal podem ser muito variados, dependendo muito da capacidade compensatória das vacas em manter a drenagem de nutrientes para o feto em crescimento.

\subsection{Formação fetal e a qualidade da progênie}

As fases do desenvolvimento fetal até o parto podem ser divididas em embrionário e fetal, cada qual possui uma série de peculiaridades e distinções. Segundo Hyttel et al. (2012), no primeiro momento ocorre a formação da maioria dos sistemas corporais, enquanto que no período de desenvolvimento fetal ocorre crescimento e maturação dos órgãos e tecidos. A nutrição materna durante a gestação pode influenciar os processos de formação e maturação dos órgãos e sistemas corporais, afetando, consequentemente, sua função fisiológica (Long et al., 2009; Reynolds et al., 2019). Os primeiros estudos sobre os insultos nutricionais durante a gestação, processo conhecido também como programação fetal, surgiram durante a Segunda Guerra Mundial quando filhos de gestantes que sofreram restrição nutricional, apesar de nascerem com peso normal, apresentaram alta incidência de doenças respiratórias, cardiovasculares e diabetes na vida adulta (Tsuneda et al., 2017).

As mudanças estruturais e funcionais dos órgãos causadas pela oferta de nutrientes durante a gestação, segundo Reynolds et al. (2019), servem para permitir uma rápida adaptação do feto em desenvolvimento à pressão de seleção ambiental uterina. Os mesmos autores afirmam que os defeitos funcionais devido à expressão gênica alterada são melhor explicados por um novo conceito denominado "epigenética", que literalmente se traduz em "acima da genética" e é definida como alterações herdáveis na expressão gênica sem alteração do código genético. Perturbações durante o desenvolvimento fetal, tais como a disponibilidade reduzida de nutrientes, podem induzir a alterações epigenéticas que promovem a formação de um fenótipo "econômico", que, quando exposto a um ambiente rico em nutrientes, tem risco aumentado de desenvolver doenças metabólicas (Vaag et al., 2012).

Essas alterações metabólicas na formação fetal estão relacionadas à saúde, sobrevivência e potencial produtivo da progênie. Webb et al. (2019) descrevem que a desnutrição ou a restrição alimentar durante a gestação acaba produzindo um fenótipo que possui maiores habilidades adaptativas quando expostos à ambientes desfavoráveis na vida adulta. Essa afirmação corrobora com Greenwood et al. (2010) os quais afirmam que animais que passaram por estresse nutricional no ambiente intrauterino possuem maior capacidade de adaptação metabólica à ambientes menos favoráveis durante a vida pós-natal, podendo apresentar ganho de peso compensatório.

A sobrevivência dos bezerros foi avaliada por Bohnert et al. (2013), os quais observaram maior percentagem de bezerros vivos ao nascimento ( $90 \%$ vs $100 \%$ ) e ao desmame (88,3\% vs 99,2\%) quando estes nasceram de vacas com maior escore corporal durante a gestação. Essas respostas demonstram que a mortalidade pós-natal é muito próxima entre filhos de vacas com melhor ou pior estado nutricional durante a gestação, sugerindo que algumas alterações metabólicas favorecem a sobrevivência dos fetos formados em ambiente uterino restrito de nutrientes. Perry et al. (2019) sugerem que a vaca pode tentar manter a transferência da imunidade passiva em face da dieta restrita de nutrientes, através da elevação na quantidade de imunoglobulinas no colostro disponível aos bezerros.

A concentração sérica de IgG foi avaliada por Bohnert et al. (2013) entre 24 e 48 horas após o nascimento dos bezerros. Os autores não observaram diferenças neste componente no sangue dos bezerros filhos de vacas com baixa ou alta condição corporal $(6,12 \mathrm{mg} / \mathrm{dL} v s 5,96 \mathrm{mg} / \mathrm{dL}$, respectivamente), sugerindo que a absorção de imunoglobulinas e a tranferência de imunidade passiva foi semelhante. Testando diferentes níveis nutricionais nos últimos terços de gestação, Duarte et al. (2013) observaram que filhos de vacas submetidas à restrição nutricional apresentam compensação na formação do trato 
digestório, mais especificamente do intestino delgado, órgão responsável pela absorção da maior parte dos nutrientes na vida inicial dos ruminantes. No referido estudo, houve aumento no comprimento tanto do intestino delgado, como das vilosidades intestinais, nos bezerros submetidos à restrição no período fetal.

A saúde e a sobrevivência inicial dos bezerros nascidos de vacas com alto ou baixo nível nutricional durante a gestação tem sido relacionada com a resposta inflamatória e à imunidade destes animais. Em sua revisão de literatura, Abuelo (2020) afirmaram que o estresse oxidativo pode afetar a resposta inflamatória e a suscetibilade da progênie à doenças. Taylor et al. (2016) avaliaram dois níveis de nutrientes digestíveis totais para vacas no segundo terço de gestação, e observaram que a progênie de vacas com status energético negativo apresentavam níveis de anticorpos mais baixos em resposta ao desafio da ovalbumina durante o período de terminação, o que pode afetar a resposta imune adaptativa destes animais.

Além da saúde, a produtividade do indivíduo é muito influenciada pela capacidade e eficiência metabólica dos órgãos envolvidos com o metabolismo animal. Segundo Symonds et al. (2010), o desfavorecimento do ambiente uterino durante a fase inicial de gestação pode resultar em alterações nos mecanismos homeostáticos do fígado e pâncreas, influenciando a capacidade da progênie em metabolizar os nutrientes. Em casos de desnutrição, o transporte de nutrientes ao feto pode ser reduzido e vice-versa nos casos de supernutrição, resultando em mudanças na disponibilidade de nutrientes (glicose, ácidos graxos e aminoácidos, por exemplo) para o feto, aspecto que finalmente pode afetar o crescimento e desenvolvimento de tecidos fetais (Gaccioli et al., 2013). Estas alterações na funcionalidade dos órgãos podem se estender até a vida adulta da prole.

A restrição de nutrientes durante a gestação precoce ou média pode afetar negativamente o desenvolvimento do pâncreas fetal (McCarty et al., 2020). O desenvolvimento do pâncreas fetal e neonatal é fundamental para controlar a homeostase da glicose durante os primeiros meses de vida. Trabalhos recentes têm demonstrado que o pâncreas bovino fetal é sensível à restrição de nutrientes no útero materno durante a gestação (Keomanivong et al., 2016). Maresca et al. (2018) observaram que bezerros nascidos de vacas com baixa ingestão de proteína durante a metade final da gestação eram hiperglicêmicos durante os primeiros 60 dias de vida mas que, após este período, as concentrações de glicose retornaram a níveis semelhantes aos de bezerros filhos de vacas com alta proteína. Os mesmos autores complementam que a alta concentração de glicose pode estar relacionada à resistência à insulina e menor atividade metabólica dos órgãos, uma vez que durante a formação embrionária o ambiente era restrito. Estes resultados sugerem que animais formados sob boa condição nutricional poderão apresentar maior eficiência na captação e aproveitamento em uma situação de alta disponibilidade de nutrientes, tais como a fase de terminação.

Apesar destas possíveis alterações na formação e saúde fetal, o potencial produtivo da prole poderá ser influenciado pela adaptação do indivíduo na vida adulta. Brameld et al. (2010) sugerem que, com tempo suficiente durante a vida pós-natal, o animal seja capaz de superar ou compensar a maioria dessas diferenças iniciais, resultando em apenas pequenos (se houver) efeitos residuais sobre a composição corporal em fases posteriores de crescimento. Ramírez et al. (2020) complementam que a restrição severa de nutrientes durante a gestação pode também compensar o crescimento do indivíduo após o nascimento, quando este for exposto à ambientes restritos também pós-natal.

\subsection{Formação do tecido muscular esquelético}

$\mathrm{Na}$ produção de bovinos de corte, o principal tecido de interesse é o muscular esquelético. Este tecido é formado basicamente por células musculares, adipócitos e fibroblastos, as quais são originadas de um mesmo conjunto de células progenitoras indiferenciadas (Du et al., 2013). Os processos de ativação, proliferação e diferenciação celular são brevemente descritos por Du et al. (2013), processos que são regulados por diversos sinalizadores genéticos e fatores de transcrição, que estimulam ou inibem a expressão dos gênes, e consequentemente, a formação de um fenótipo específico. De acordo com Du et 
al. (2010), a formação do tecido muscular ocorre pelos processos de hiperplasia e hipertrofia das fibras musculares, sendo a primeira dividida em miogênese primária e secundária. Os autores afirmam que a miogênese primária tem início nos primeiros meses de gestação, onde ocorre a formação das fibras primárias, as quais servirão de suporte para as fibras secundárias formadas entre o segundo e oitavo mês de gestação.

A hipertrofria das fibras musculares pré-existentes tem início entre o quinto e sexto mês de gestação e ocorre juntamente com a adipogênese intramuscular e o desenvolvimento final dos órgãos e sistemas corporais (Du, et al., 2010). A hipertrofia muscular caracteriza-se pela proliferação das células satélites existentes entre a membrana plasmática das fibras musculares e a lâmina basal. Quando estimuladas, as células satélites proliferam e se fundem com as fibras pré-existentes, e começam a sintetizar proteínas que resultam em aumento no volume muscular através da formação de novos sarcômeros (Mendes, 2016). Caso houver baixo número de fibras musculares formadas no período fetal, o crescimento muscular será limitado, uma vez que as fibras musculares não podem exceder o tamanho que possibilite a troca eficiente de nutrientes e metabólitos (Du et al., 2013).

A formação do tecido muscular, assim como os demais sistemas corporais, pode ser influenciada pela densidade de nutriente que chega ao ambiente uterino. Diante do exposto, Du et al. (2010) afirmam que a restrição nutricional durante os dois primeiros terços de gestação reduz o número de fibras musculares e, consequentemente, a massa muscular da progênie. Os autores complementam que se a restrição de nutrientes ocorrer no terço final da gestação, os processos de hipertrofia das fibras musculares e da adipogênese intramuscular serão comprometidos, podendo reduzir, respectivamente, o peso ao nascer da progênie e a gordura de marmoreio na carne dos descendentes.

Os processos de miogênese, adipogênese e fibrogênese estão mais suscetíveis aos efeitos da restrição de nutrientes durante o período gestacional. Zhu et al. (2006) afirmam que durante a gestação o músculo esquelético possui menor prioridade na partição de nutrientes do feto em relação aos órgãos vitais tais como cérebro, coração e fígado. Estudando os efeitos da restrição alimentar nos primeiros dois terços de gestação, Long et al. (2009) observaram maior peso relativo do coração e cérebro aos 125 dias de gestação em fetos submetidos à restrição alimentar em relação aos filhos de vacas com melhor nutrição, indicando que o desenvolvimento do músculo esquelético possui menor prioridade em condições de baixa oferta de nutrientes.

A adipogênese intramuscular, conforme Du et al. (2010), ocorre com maior intensidade no terço final de gestação, com um declíneo do potencial adipogênico após o nascimento. A maior formação de adipócitos intramusculares normalmente está associada à maior oferta de nutrientes para a fêmea no terço final de gestação, podendo haver diferentes respostas na qualidade final da carne. A supernutrição das ovelhas durante o final da gestação não afetou a gordura dos fetos de cordeiros, mas, houve aumento na expressão de genes associados à adipogênese e lipogênese no tecido adiposo (Reynolds et al., 2019). Do mesmo modo, Greenwood et al. (2010) afirmam que a restrição nutricional diminui a deposição de tecido adiposo na carcaça, devido principalmente à menor diferenciação celular e formação de adipócitos. A redução da adipogênese no final de gestação poderá resultar em maior formação de tecido conjuntivo, uma vez que estes processos se originam do mesmo pool de células multipotentes (Du et al., 2015). Nessa situação, pode haver redução na maciez da carne.

Porém, carcaças com maior teor de gordura também podem ser obtidas quando os animais forem gestados em ambientes com baixa oferta de nutrientes. Mohrhauser et al. (2015b) afirmam que a mudança na distribuição adiposa pode ser consistente com a teoria do feto desenvolvendo um fenótipo "econômico" devido à inadequada disponibilidade de nutrientes. Segundo os autores, o feto se prepara para um ambiente de nutrientes escassos criando uma composição corporal mais eficiente, com maior deposição intramuscular de gordura para aumentar armazenamento de energia dentro do músculo. Assim, essa energia de reserva poderia teoricamente ser facilmente utilizada por células musculares quando necessário. Outra teoria 
foi apresentada por Brameld et al. (2010), os quais hipotetizaram que a formação dos adipócitos pode ser uma função padrão das células, caso estas não forem estimuladas para outras funções durante a gestação.

Como vimos, a composição do músculo esquelético poderá ser influenciada ou modulada pela nutrição materna durante a gestação. Contudo, o grau de interferência nutricional vai depender do genoma individual de cada raça. Chen et al. (2019) estudou a expressão de genes em raças com alto e baixo potencial de marmorização da carne e observaram que o grupo de animais com mais gordura intramuscular na carne apresentou maior expressão de genes relacionados com a adipogênese, enquanto que genes fibrogênicos e miogênicos foram se expressar mais no grupo de baixo marmoreio no músculo Longissimus dorsi. Duarte et al. (2013) também obtiveram maior expressão de genes miogênicos em bovinos da raça Aberdeen Angus em comparação à Wagyu, demonstrando que o potencial genético do indivíduo pode definir o grau de influência da nutrição materna durante a gestação sobre a formação do músculo esquelético fetal.

\subsection{Desempenho e características pós-abate da progênie}

As respostas produtivas da progênie aos insultos nutricionais durante a fase de formação fetal são ainda muito controversas. Além da interferência do genoma do indivíduo, a magnitude de qualquer efeito da nutrição parece depender do nível de insulto nutricional à vaca gestante, do momento desse insulto durante o estágio de desenvolvimento fetal, e da idade em que a prole é estudada após o nascimento (Brameld et al., 2010). Além disso, a produtividade dos descendentes pode estar relacionada às inúmeras alterações fisiológicas e estruturais dos órgãos corporais, as quais somadas à melhor formação do tecido muscular, por exemplo, poderá resultar em aumento na produtividade destes animais na vida pós-natal.

Diversos estudos avaliaram os efeitos da nutrição materna durante a gestação sobre a produtividade da progênie na vida adulta, divergindo entre eles principalmente quanto aos níveis de restrição alimentar, nutriente restrito (proteína e/ou energia) e período gestacional da deficiência nutricional. Nossa revisão teve maior foco na nutrição da vaca durante a metade final de gestação, uma vez que alterações no crescimento pós-natal são mais pronunciadas se a desnutrição materna englobar o final do período gestacional (Greenwood et al., 2019). Testando os efeitos dos níveis de proteína na metade final de gestação, LeMaster et al. (2017) observaram maior peso ao nascer dos bezerros filhos de vacas submetidas à dieta para mantença em relação às vacas restritas. Estes resultados diferem de Maresca et al. (2018) os quais forneceram 64\% e 121\% das exigências de proteína no final da gestação e não observaram efeitos no peso ao nascimento da progênie.

O desempenho da prole durante a fase de terminação em confinamento foi avaliado por Mulliniks et al. (2013), os quais testaram diferentes níveis de suplementação proteica durante o final da gestação. Estes autores não verificaram alterações no desempenho e nas características da carcaça e carne da progênie. Esses resultados corroboram com Maresca et al. (2019), os quais testaram 64 e $121 \%$ de proteína bruta nos dois últimos terços de gestação e não observaram alterações no desempenho da prole durante as fases de crescimento e terminação. Porém, os autores obtiveram maior área de Longissimus dorsi na progênie de vacas alimentadas com alto teor de proteína na gestação $\left(63,74\right.$ e $69,39 \mathrm{~cm}^{2}$, respectivamente). Esse resultado pode ser reflexo do favorecimento dos processos de miogênese no período fetal das vacas que receberam maior nível de proteína. As características da carne também foram influenciadas pela restrição proteica ( 80 vs $108 \%$ das exigências), principalmente quando este desafio nutricional ocorreu no segundo terço de gestação (Webb et al., 2019). Os autores observaram maior teor de gordura, e redução na umidade e na força de cisalhamento da carne de novilhos nascidos de vacas com maior teor de proteína na dieta.

Os efeitos do nível energético na dieta de vacas gestantes também vem sendo amplamente estudado. Wilson et al. (2016) testaram o fornecimento de 100 ou $125 \%$ das exigências de energia no final de gestação e obtiveram maior peso ao nascimento dos bezerros filhos de vacas com maior ingestão de energia. Os mesmos autores não observaram diferenças no desempenho pós-natal e nas características da carcaça e carne da progênie. A restrição energética no segundo terço de gestação 
foi testada por Taylor et al. (2016), os quais não observaram diferenças tanto no peso ao nascer, como no desempenho pós natal dos filhos de vacas submetidas a status energético negativo (80\% das exigências) ou para mantença de condição corporal neste período gestacional. Mohrhauser et al. (2015a) não observaram alterações nas características de carcaça dos filhos de vacas que receberam 80 ou $100 \%$ dos requerimentos de energia no segundo terço da gestação, demonstrando que as vacas podem se adaptar e compensar a partição de nutrientes ao feto em situações de restrições nutricionais pouco severas.

Os efeitos do status energético da vaca no terço final de gestação (50, 75 ou $100 \%$ das exigências de mantença) foram testados por Ramírez et al. (2020). Os autores observaram aumento linear no peso ao nascer dos bezerros à medida que melhorou o nível de energia da vaca neste período gestacional (31,5; 34,6 e 36,8 kg, respectivamente). Porém, filhos de vacas que receberam apenas $50 \%$ das exigências energéticas apresentaram um ganho de peso compensatório após o nascimento, resultando em maior peso de abate em relação aos filhos de vacas com 75\% dos requerimentos. Mesmos comportamentos foram observados para a espessura de gordura subcutânea no referido estudo (7,1; 5,0 e 6,6 mm, respectivamente para 50, 75 e $100 \%$ das exigências). Os autores justificam o ganho compensatório e maior desempenho pós-natal dos bezerros nascidos de vacas com restrição severa, à formação de um fenótipo "econômico" com maior capacidade de adaptação às condições de menor oferta de nutrientes após o nascimento.

A avaliação do escore de condição corporal pode ser considerada um dos melhores métodos para prever o status nutricional do rebanho. Bohnert et al. (2013) agruparam as vacas de acordo com o escore corporal na gestação, e verificaram que vacas com maiores reservas corporais produziram bezerros mais pesados ao nascimento (38,8 vs 41,4 kg). Porém, o desempenho da progênie na vida adulta e as características de carcaça foram semelhantes entre os filhos de vacas com baixa ou alta condição corporal na gestação. Os efeitos da condição corporal das vacas na gestação também foram avaliados por Marques et al. (2016), os quais observaram maior peso ao desmame dos filhos de matrizes que ganharam escore corporal no segundo ou terceiro trimestre de gestação, em relação às alimentadas para ganhar condição corporal no início da gestação ou para manter o escore adequado e inadequado durante toda a gestação.

Os resultados apresentados nesta revisão sugerem que os efeitos da nutrição materna na gestação podem ser mais facilmente constatados nos meses inicias de vida da progênie. Além disso, estes efeitos tendem a desaparecer com o avançar da idade dos indivíduos em resposta à maior adaptação pós-natal dos animais formados em um ambiente uterino restrito de nutrientes, os quais normalmente apresentam um ganho de peso compensatório na vida adulta.

\section{Considerações Finais}

A restrição nutricional durante a gestação promove adaptações na partição de nutrientes para o feto. O ambiente intrauterino restrito resulta em alterações na fisiologia e função dos órgãos fetais formando um fenótipo "econômico" capaz de produção compensatória na vida pós-natal.

O tecido muscular esquelético possui menor prioridade na partição de nutrientes no organismo fetal e um ambiente de restrição nutricional durante a gestação interfere negativamente na sua formação.

Os efeitos da nutrição materna durante a gestação sobre a produtividade da progênie apresentam divergências entre os nutrientes restritos, o período de restrição alimentar e as características avaliadas. Contudo, mudanças no desempenho da prole são mais frequentes nos meses iniciais de vida e tendem a desaparecer com o avançar da idade do indivíduo. 


\section{Referências}

Abuelo, A. (2020). Symposium review: Late-gestation maternal factors affecting the health and development of dairy calves. Journal of Dairy Science, 103(4), 3882-3893.

Bauman, D. E., \& Currie, B. (1980). Partitioning of nutrients during pregnancy and lactation: a review of mechanisms involving homeostasis e homeorhesis. Journal of Dairy Science, 63(9), 1514-1529.

Bohnert, D. W., Stalker, L. A., Mills, R. R., Nyman, A., Falck, S. J., \& Cooke, R. F. (2013). Late gestation suplementation of beff cows differing in body condition score: Effects on cow and calf performance. Journal of Animal Science, 91(11), 5485-5491.

Brameld, J. M., Greenwood, P. L., \& Bell, A. W. (2010). Biological Mechanisms of Fetal Development Relating to Postnatal Growth, Efficiency and Carcass Characteristics in Ruminants. Greenwood et al. (ed). Managing the prenatal environment to enhance livestock productivity. Dordrecht: Springer Science and Business Media, cap. 4, p. 93-120.

Camacho, L. E., Lemley, C. O., Dorsam, S. T., Swanson, K. C., \& Vonnahme, K. A., (2018). Effects of maternal nutrient restriction followed by realimentation during early and mid-gestation in beef cows. II. Placental development, umbilical blood flow, and uterine blood flow responses to diet alterations. Theriogenology, 116, 1-11.

Chen, D., Li, W., Du, M., \& Cao, B. (2019). Adipogenesis, fibrogenesis and myogenesis related gene expression in longissimus muscle of high and low marbling beef cattle. Livestock Science, 229, 188-193.

Du, M., Tong, J., Zhao, J., Underwood, K. R., Zhu, M., Ford, S. P., \& Nathanielsz, P. W. (2010). Fetal programming of skeletal muscle development in ruminant animals. Journal Animal Science, 88, 51-60.

Du, M., Huang, Y., Das, A. K., Yang, Q., Duarte, M. S., Modson, M. V., \& Zhu, M. J. (2013). Manipulating mesenchymal progenitor cell differentiation to optimize performance and carcass value of beef cattle. Journal Animal Science, 91(3), 1419-1427.

Du, M., Wang, B., Fu, X., Yang, Q., \& Zhu, M. (2015). Fetal programming in meat production. Meat Science, 109, $40-47$.

Duarte, M. S., Gionbelli, M. P., Paulino, P. V. R., Serão, N. V. L., Martins, T. S., Tótaro, P. I. S., Neves, C. A., Valadares Filho, S. C., Dodson, M. V., Zhu, M., \& Du, M. (2013). Effects of maternal nutrition on development of gastrointestinal tract of bovine fetus at different stages of gestation. Livestock Science, $153,60-65$.

Gaccioli, F., Lager, S., Powell, T. L., \& Jansson, T. (2013). Placental transport in response to altered maternal nutrition. Journal of Developmental Origins of Health and Disease, 4, 101-115.

González-Recio, O., Ugarte, E.; \& Bach, A. (2012). Trans-Generational Effect of Maternal Lactation during Pregnancy: A Holstein Cow Model. Plos One, $7(12), 1-7$.

Greenwood, P. L., Thompsom, A. N., Ford, S. P. (2010). Posnatal consequences of the maternal environment and growth during prenatal life for productivity of ruminants. Greenwood et al. (ed). Managing the prenatal environment to enhance livestock productivity. Dordrecht: Springer Science and Business Media, cap. 1, p. 3-36.

Greenwood, D. P., \& Bell, A. W. (2019). Developmental programming and growth of livestock tissues for meat production. Veterinary Clinics Food Animal, $35,303-319$.

Hyttel, P., Sinowatz, F., \& Vejlsted, M. (2012). Embriologia veterinária. Rio de Janeiro: Elsevier.

Keomanivong, F. E., Camacho, L. E., Lemley, C. O., Kuemper, E. A., Yunusova, R. D., Borowicz, P. P., Vonnahme, K. A., Caton, J. S., \& Swanson, K. C. (2016). Effects of realimentation after nutrient restriction during mid- to late gestation on pancreatic digestive enzymes, serum insulin and glucose levels, and insulin-containing cell cluster morphology. Animal Phisiology and Animal Nutrition, 101(3), 589-604.

LeMaster, C. T., Taylor, R. K., Ricks, R. E., \& Long, N. M. (2017). The effects of late gestation maternal nutrient restriction whit or without protein supplementation on endocrine regulation of newborn and postnatal beef calves. Theriogenology, 87, 64-71.

Long, N. M., Vonnahme, K. A., Hess, B. W., Nathanielsz, P. W., \& Ford, S. P. (2009). Effects of early gestational undernutrition on fetal growth, organ development, and placentomal composition in the bovine. Journal of Animal Science, 87, 1950-1959.

Maresca, S., Lopes Valiente, S., Rodrigues, A. M., Long, N. M., Pavan, E., \& Quintans, G. (2018). Effect of protein restriction of bovine dams during late gestation on offspring postnatal growth, glucose-insulin metabolism and IGF-1 concentration. Livestock Science, 212, $120-126$.

Maresca, S., López Valiente, S., Rodriguez, A. M., Testa, L. M., Long, N. M., Quintans, G. I., Pavan, E. (2019). The influence of protein restriction during mid- to late gestation on beef offspring growth, carcass characteristic and meat quality. Meat Science, 153, $103-108$.

Marques, R. S., Cooke, R. F., Rodrigues, M. C., Moriel, P., \& Bohnert, D. W. (2016). Impacts of cow body condition score during gestation on weaning performance of the offspring. Livestock Science, 191, 174-178.

McCarty, K. J., Washburn, J. L., Taylor, R. K., \& Long, N. M. (2020). The effects of early or mid-gestation nutrient restriction on bovine fetal pancreatic development. Domestic Animal Endocrinology, 70, 1-6.

Mendes, L. C. M. (2016). Trabalho de Conclusão de Curso em Zootecnia - Universidade Federal do Rio Grande do Sul, Porto Alegre, RS.

Mohrhauser, D. A., Taylor, A. R., Underwood, K. R., Pritchard, R. H., Wertz-Lutz, A. E., \& Blair, D. A. (2015a). The influence of maternal energy status during mid-gestation on beef offspring tenderness, muscle characteristics, and gene expression. Meat Science, 110, $201-211$. 
Mohrhauser, D. A., Taylor, A. R., Underwood, K. R., Pritchard, R. H., Wertz-Lutz, A. E., \& Blair, D. A. (2015b). The influence of maternal energy status during midgestation on beef offspring carcass characteristics and meat quality. Journal of Animal Science, 93, 786-793.

Muhlhauser, B. S., Duffield, J. A., \& McMillen I. C. (2007). Increased Maternal Nutrition Increases Leptin Expression in Perirenal and Subcutaneous Adipose Tissue in the Postnatal Lamb. Endocrinology, 148, (12), 6157-6163.

Mulliniks, J. T., Mathis, C. P., Cox, S. H., \& Petersen, M. K. (2013). Supplementation strategy during late gestation alters steer progeny health in the feedlot without affectingcow performance. Animal Feed Science and Technology, 185, 126-132.

Perry, V. E. A., Copping, K. J., Miguel-Pacheco, G., \& Hernandez-Mendrano, J. (2019). The effects of developmental programming upon neonatal mortality. Veterinary Clinics Food Animal, 35, 289-302.

Ramírez, M., Testa, L. M., López Valiente, S., Latorre, M. E., Long, N. M., Rodriguez, A. M., Pavan, E., \& Maresca, S. (2020). Maternal energy status during late gestation: Effects on growth performance, carcass characteristics and meat quality of steers progeny. Meat Science, $164,1-7$.

Reynolds, L. P., Borowicz, P. P., Caton, J. S., Crouse, M. S., Dahlen C. R., \& Ward, A. K. (2019). Developmental Programming of Fetal Growth and Development. Veterinary Clinics Food Animal, 35(2), 229-247.

Symonds, M. E., Sebert, S. P., \& Budge, H. (2010). Nutritional regulation of fetal growth and implications for productive life in ruminants. Animal, 4(7), 1075-1083.

Taylor, A. R., Mohrhauser, D. A., Pritchard, R. H., Underwood, K. R., Wertz-Lutz, A. E., \& Blair, D. A. (2016). The influence of maternal energy status during mid-gestation on growth, cattle performance, and the immune response in the resultant beef progeny. The Professional Animal Scientist, 32 , 389-399.

Tsuneda, P. P., Hatamoto- Zervoudakis, L. K., Duarte Júnior, M. F., Silva, L. E. S., Delbem, R. A., \& Motheo, T. F. (2017). Efeitos da nutrição materna sobre o desenvolvimento e performance reprodutiva da prole de ruminantes. Investigação, 16(1), 56-61.

Vaag, A. A., Grunnet, L. G., Arora, G. P., \& Brons, C. (2012). The thrifty phenotype hypothesis revisited. Diabetologia, 55, 2085-2088.

Zhu, M. J., Ford, S. P., Means, W. J., Hess, B. W., Nathaniels, P. W., \& Du, M. (2006). Maternal nutrient restriction affects properties of skeletal muscle in offspring. The Journal of Physiology, 575(1), 241-250.

Webb, M. J., Block, J. J., Funstons, R. N., Underwood, K. R., Legako, J. F., Harty, A. A., Slaverson, R. R., Olson, K. C., \& Blair, A. D. (2019). Influence of maternal protein restriction in primiparous heifers during mid and/or late-gestation on meat quality and fatty acid profile of progeny. Meat Science, $152,31-37$.

Wilson, T. B., Faulkner, D. B., \& Shike, D. W. (2016). Influence of prepartum dietary on beef cow performance and calf growth and carcass characteristics. Livestock Science, 184, 21-27. 